\title{
Analysis of soil physico-chemical properties of guava orchard in multi zones of district Larkana, Sindh, Pakistan
}

Muhammad Mukhtar Rajput ${ }^{1}$, Allah Wadhayo Gandahai ${ }^{1}$, Mehrunisa Memon $^{1}$, Arshad Ali Kaleri², Mukesh Kumar Soothar ${ }^{1 *}$, Sajid Hussain Kaleri ${ }^{1}$, Mahendar Kumar Soothar ${ }^{1}$ and Ayaz Ali Panhwar ${ }^{1}$

1. Department of Soil Science, Sindh Agriculture University Tandojam-Pakistan

2. Department of Plant Breeding and Genetics, Sindh Agriculture University Tandojam-Pakistan

*Corresponding author's email: mukeshksootar@gmail.com

\section{Citation}

Muhammad Mukhtar Rajput, Allah Wadhayo Gandahai, Mehrunisa Memon, Arshad Ali Kaleri, Mukesh Kumar Soothar, Sajid Hussain Kaleri, Mahendar Kumar Soothar and Ayaz Ali Panhwar. Analysis of soil physicochemical properties of guava orchard in multi zones of district Larkana, Sindh, Pakistan. Pure and Applied Biology. Vol. 6, Issue 3, pp841-849. http://dx.doi.org/10.19045/bspab.2017.60088

\begin{tabular}{llll}
\hline \hline Received: 15/03/2017 & Revised: 15/06/2017 & Accepted: 02/07/2017 & Online First: 06/07/2017 \\
\hline \hline
\end{tabular}

\section{Abstract}

This study was an effort to assess the soil physico-chemical properties of guava orchards in four Taluka of district Larkanaduring 2014-15. Composite soil samples were collected from three (4x4 each) blocks (16 tress) of each orchard at three soil depths $(0-15,15-30$ and 30-45 cm). The soil samples were analyzed for some physical and chemical properties of the soil. Analytical results of this study described that soils of taluka Bakrani and Dokri orchards were classified as silty clay and Larkana and Ratodero orchards had silty clay loam texture. Electrical conductivity of soil samples of guava orchards in four taluka of district Larkana, showed that $100 \%$ of the soils of study area has an average value of $\left(<1 \mathrm{dsm}^{-}\right.$ $\left.{ }^{1}\right)$. For $\mathrm{pH}$, however, all soil samples were found medium alkaline ( $\left.\mathrm{pH} 7.6-8.3\right)$. In Pakistan, guava is grown on an area of 64.0 hectares with a total production of 546.6 tons. Sindh contributes 9.8 hectares of area under guava with a total production of 75.4 tons (GOP, 2012). In case of organic matter, results of this study revealed that all soil samples were found poor $(<0.86)$ in OM content except surface soil $(0$ $15 \mathrm{~cm}$ ) of Bakrani guava orchards. All the guava orchard soils were put in the categorization of very marginal calcareous $(5-10 \%)$. Results regarding soluble $\mathrm{Na}^{+}$showed that average soluble $\mathrm{Na}^{+}$contents ranged between $0.30-0.45$ meq L $\mathrm{L}^{-1}$ across the four taluka's of Larkana. Overall results showed that $100 \%$ soil samples were found marginal in soluble $\mathrm{Na}^{+}$. Soluble $\mathrm{Ca}^{2+}$ ranged between 5.37 to 8.74 meq $\mathrm{L}^{-1}$. Ratodero guava orchard soils have more $\mathrm{Ca}^{2+}$ content than other taluka's followed by Larkana. Further study revealed that $100 \%$ of the samples were found marginal in $\mathrm{Ca}^{2+}$ content. Similarly, $100 \%$ of the soil samples were marginal in $\mathrm{Mg}$ content. In this study $\mathrm{K}$ ranged between 302-403 with an average $\mathrm{K}$ of 351 meq $\mathrm{L}^{-1}$ across four taluka.

Keywords: Physico-chemical properties; Organic matter; Calcium carbonate; Potassium in soil

\section{Introduction}

Orchard soils under semiarid/arid subtropical regions have low organic carbon content and inherent low soil fertility status [1]. Poor soil fertility seems to be one of the most important reasons for low fruit productivity [2]. Restoration of the soils are important if tree crops are planted from view point of orchard sustainability and productivity as millions of farmers are 
engaged in fruit production, sustaining their livelihood and nutritional security [3]. The environmental condition of Pakistan is good for the cultivation of different varieties of vegetables and fruits. Fruits contribute for the production of drinks [4]. Among fruits guava has importance in Pakistan fruit industry and it is ranked as $4^{\text {th }}$ place on the basis of area (62.5 thousand ha) and production (555 thousand tons) [5]. Sindh contributes 9.8 hectares of area under guava with a total production of 75.4 tons [6]. It has a high nutritional importance. It produces the fruit two times in a year i.e. Kharif (summer) and Rabi (winter) however the most excellent fruit is obtained in winter $[7,8]$ conducted experiment based on the nutrient requirement of guava trees. [9] determined guava leaves samples and observed NPK and Mg and Zn deficiency in the orchards. Guava is very good source of vitamin $\mathrm{C}$ when compared with citrus and apple [10]. Punjab province shares $77 \%$ of guava production in the Pakistan [11]. The taking away of nutrients from guava orchards happens because of the harvested fruit, as was stated earlier, and also by pruning the trees. In adult guava orchards, very much pruning is practiced, which may greatly cause in decrease aboveground biomass to 40 to $60 \%$. The scraped material consists of about $24.5 \mathrm{~kg}$ of green material per plant $(7.8 \mathrm{~kg}$ of leaves; $2 \mathrm{~kg}$ of boughs and $14.7 \mathrm{~kg}$ of branches, twigs and small fruits [12]. Locally, number of guava trees is found unhealthy and premature death is common problem. As a result very little knowledge is available about phyicochemical properties of the soil of guava orchard's [13]. Therefore soil should be analyzed for macro and micro nutrients for improving tree health, fruit yield and quality [14]. Soil fertility is decreasing on daily basis because of intensive cropping to fulfill the needs of rapidly rising population [14]. Therefore, this study is proposed to explore orchards of district Larkana, Sindh for their physico-chemical properties.

\section{Materials and methods}

This study was conducted to investigate soil physico-chemical properties of guava orchards soil of district Larkana, Sindh. Soil sampling was done to collect samples. The collected soil samples were analyzed for particle size distribution by Hydrometer method, EC and $\mathrm{pH}$ were determined by digital $\mathrm{EC}$ and $\mathrm{pH}$ meter, calcium carbonate, and organic matter was determined by Walkley and Black method, soluble $\mathrm{Na}^{+}, \mathrm{K}^{+}$ were analyzed by ammonium acetate method, extractable $\mathrm{Ca}^{+}$and $\mathrm{Mg}^{+}$were analyzed by ammonium acetate and soluble $\mathrm{Cl}^{-}$was determined by Mohar's titration.

\section{Results and discussion}

The result of particle size distribution analysis of guava orchard soils of all Taluka at different soil depths is presented in Figure 1. The data showed a sand percent of 8.8 , $15.3,7.29$ and 14.1, silt percent of 42.8, 41.1, 36.6, and 36.9 and clay 48.4, 43.6, 56.11 and $49 \%$ in taluka Bakrani and Dokri, Larkana and Ratodero were classified respectively. The soils of Taluka Bakrani and Dokri orchards were classified as silty clay and Larkana and Ratodero orchards had silty clay loam in texture.

Electrical conductivity $\left(\mathbf{d S} \mathbf{m}^{-\mathbf{1}}\right)$

Result for electrical conductivity (EC) of soil samples of guava orchards in four taluka of district Larkana, (Table 1) shows that there is little different in EC of soil at various depths. Higher EC at all depths across taluka values were observed at surface soil than subsurface. However, all soil samples were noted non-saline $\left(<2 \mathrm{dsm}^{-}\right.$ ${ }^{1}$ ) as categorized by Jackson [15]. 


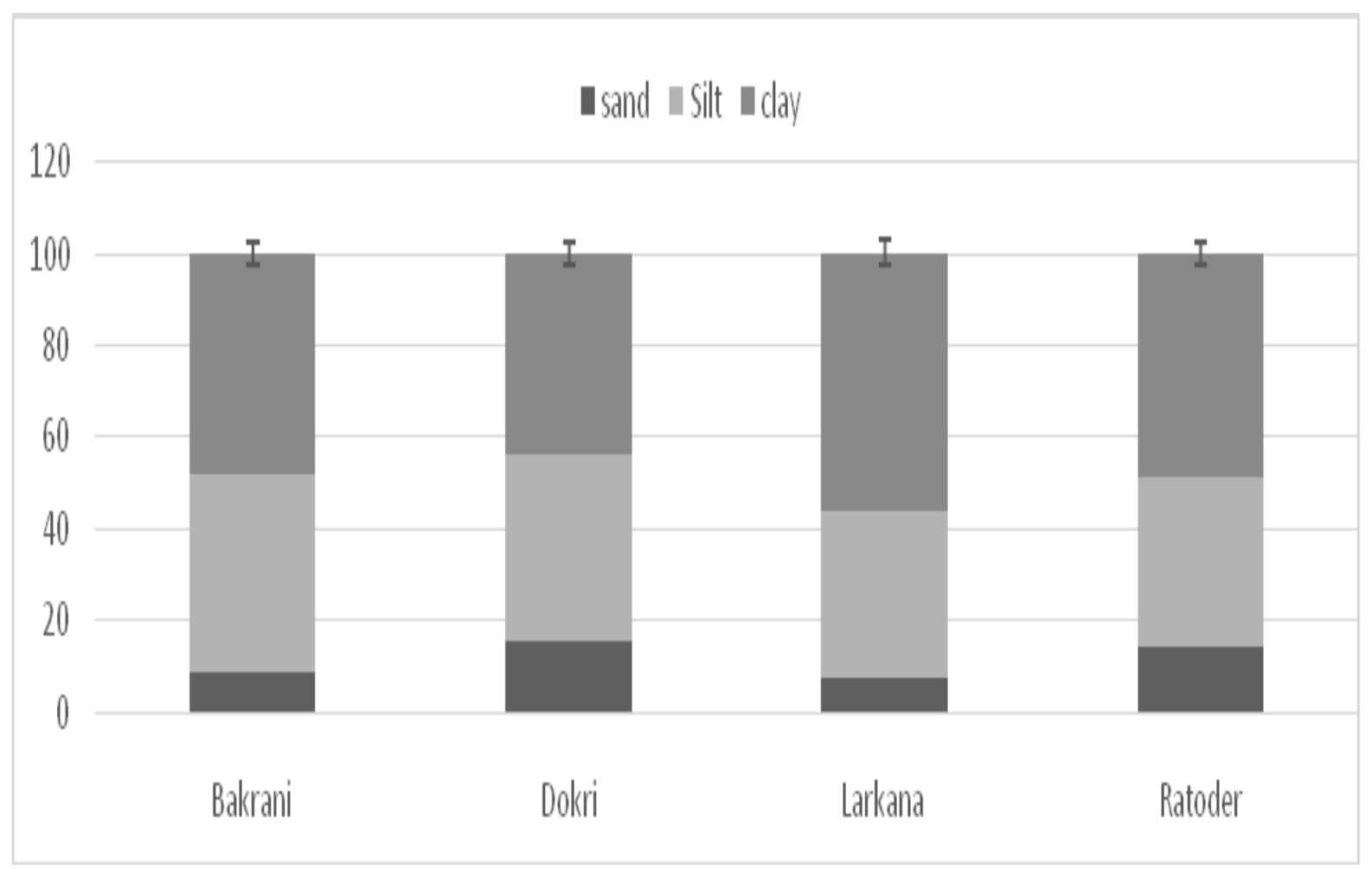

Figure 1. Particle size distribution (\%) of four Talukas of guava orchards of district Larkana, Sindh

Table 1. Electrical conductivity $\left(\mathrm{dS} \mathrm{m}^{-1}\right)$ in guava orchard soils of districtLarkana, Sindh

\begin{tabular}{|c|c|c|c|c|c|c|c|}
\hline \multirow[b]{2}{*}{ Taluka } & \multirow[b]{2}{*}{$\begin{array}{l}\text { Soil } \\
\text { depth } \\
(\mathrm{cm})\end{array}$} & \multirow[b]{2}{*}{ Range } & \multirow[b]{2}{*}{ Average } & \multirow{2}{*}{$\begin{array}{l}\text { Mean } \pm \\
\text { standard } \\
\text { deviation }\end{array}$} & \multicolumn{3}{|c|}{ Categorization (sample \%) } \\
\hline & & & & & $\begin{array}{l}\text { Non- } \\
\text { saline } \\
(<2) \\
\end{array}$ & $\begin{array}{l}\text { Slightly } \\
\text { Saline } \\
(2-4) \\
\end{array}$ & $\begin{array}{l}\text { Moderately } \\
\text { Saline } \\
(4-8) \\
\end{array}$ \\
\hline \multirow{3}{*}{ Bakrani } & $0-15$ & $0.30-0.62$ & 0.46 & $0.46 \pm 0.23$ & $100 \%$ & - & - \\
\hline & $15-30$ & $0.22-0.50$ & 0.36 & $0.36 \pm 0.20$ & $100 \%$ & - & - \\
\hline & $30-45$ & $0.16-0.43$ & 0.29 & $0.29 \pm 0.19$ & $100 \%$ & - & - \\
\hline \multirow{3}{*}{ Dokri } & $0-15$ & $0.44-0.89$ & 0.66 & $0.66 \pm 0.32$ & $100 \%$ & - & - \\
\hline & $15-30$ & $0.31-0.6$ & 0.45 & $0.45 \pm 0.20$ & $100 \%$ & - & - \\
\hline & $30-45$ & $0.2-0.53$ & 0.36 & $0.36 \pm 0.22$ & $100 \%$ & - & - \\
\hline \multirow{3}{*}{ Larkana } & $0-15$ & $0.3-0.6$ & 0.45 & $0.45 \pm 0.21$ & $100 \%$ & - & - \\
\hline & $15-30$ & $0.2-0.53$ & 0.36 & $0.36 \pm 0.23$ & $100 \%$ & - & - \\
\hline & $30-45$ & $0.18-0.52$ & 0.35 & $0.35 \pm 0.24$ & $100 \%$ & - & - \\
\hline \multirow{3}{*}{$\begin{array}{l}\text { Ratoder } \\
\text { o }\end{array}$} & $0-15$ & $0.24-0.72$ & 0.48 & $0.48 \pm 0.34$ & $100 \%$ & - & - \\
\hline & $15-30$ & $0.21-0.56$ & 0.38 & $0.38 \pm 0.25$ & $100 \%$ & - & - \\
\hline & $30-45$ & $0.25-0.57$ & 0.41 & $0.41 \pm 0.23$ & $100 \%$ & - & - \\
\hline
\end{tabular}


pH

Results regarding $\mathrm{pH}$ is presented in Table 2. The average soil $\mathrm{pH}$ of all taluka was recorded as (8.21), whereas minimum $\mathrm{pH}$ value was found (7.78) and maximum $\mathrm{pH}$ of (8.88).As compared to other larkana taluka orchards soil has more average $\mathrm{pH}$ (8.74). Whereas, Bakrani (7.92) and Ratodero (7.96) has lowest $\mathrm{pH}$ among all four studied talukas.

Table 2. pH in guava orchard soils of district Larkana, Sindh

\begin{tabular}{|c|c|c|c|c|c|c|c|}
\hline \multirow[b]{2}{*}{ Taluka } & \multirow[b]{2}{*}{$\begin{array}{l}\text { Soil } \\
\text { depth } \\
\text { (cm) }\end{array}$} & \multirow[b]{2}{*}{ Range } & \multirow[b]{2}{*}{ Average } & \multirow[b]{2}{*}{$\begin{array}{l}\text { Mean } \pm \\
\text { standard } \\
\text { deviation }\end{array}$} & \multicolumn{3}{|c|}{ Categorization (sample \%) } \\
\hline & & & & & $\begin{array}{l}\text { Slightly } \\
\text { Alkaline } \\
(\mathbf{7 . 1 - 7 . 5 )}\end{array}$ & $\begin{array}{l}\text { Medium } \\
\text { Alkaline } \\
(\mathbf{7 . 6 - 8 . 3 )}\end{array}$ & $\begin{array}{l}\text { Strongly } \\
\text { Alkaline } \\
(8.4-9.0)\end{array}$ \\
\hline \multirow{3}{*}{ Bkrani } & $0-15$ & $7.6-7.9$ & 7.75 & $7.75 \pm 0.21$ & - & $100 \%$ & - \\
\hline & $15-30$ & $7.8-8$ & 7.9 & $7.9 \pm 0.07$ & - & $100 \%$ & - \\
\hline & $30-45$ & $8.0-8.1$ & 8.05 & $8.05 \pm 0.07$ & - & $100 \%$ & - \\
\hline \multirow{3}{*}{ Dokri } & $0-15$ & $7.6-8.74$ & 8.17 & $8.17 \pm 0.81$ & - & $80 \%$ & $20 \%$ \\
\hline & $15-30$ & $7.8-8.8$ & 8.3 & $8.3 \pm 0.71$ & - & $80 \%$ & $20 \%$ \\
\hline & $30-45$ & $7.9-8.9$ & 8.4 & $8.4 \pm 0.71$ & - & $70 \%$ & $30 \%$ \\
\hline \multirow{3}{*}{ Larkana } & $0-15$ & $8.2-9.0$ & 8.6 & $8.6 \pm 0.56$ & - & $10 \%$ & $90 \%$ \\
\hline & $15-30$ & $8.5-8.9$ & 8.7 & $8.7 \pm 0.21$ & - & - & $100 \%$ \\
\hline & $30-45$ & $8.6-9.0$ & 8.8 & $8.8 \pm 0.21$ & & - & $100 \%$ \\
\hline \multirow{3}{*}{ Ratodero } & $0-15$ & $7.6-8$ & 7.8 & $7.8 \pm 0.21$ & - & $100 \%$ & - \\
\hline & $15-30$ & $7.8-8.1$ & 7.95 & $7.95 \pm 0.21$ & - & $100 \%$ & - \\
\hline & $30-45$ & $7.9-8.3$ & 8.1 & $8.1 \pm 0.28$ & - & $100 \%$ & - \\
\hline
\end{tabular}

\section{Organic matter (\%)}

The organic matter (OM) content of soil samples of guava orchards in all taluka of district Larkana. (Table 3) showed that there is slightly variation in organic matter content of soil at different soil depths. The Highest organic matter content was found at surfaceas compared to subsurface soil. All soil samples were found poor $(<0.86)$ in O.M content as categorized by Walkley and Black [16] except surface soil $(0-15 \mathrm{~cm})$ of Bakrani guava orchards. The lowest OM content was observed in Larkana guava orchard soils which ranged between 0.22 and $0.56 \%, 0.38$ and 0.70 and 0.59 and $0.84 \%$ with average contents of $0.40,0.51$ and 0.69 at $0-15,15-30$ and $30-45 \mathrm{~cm}$ soil depth, respectively. [17] evaluated the effect of farm yard manure and inorganic fertilizers applied alone or in combination on the productivity of winter crop of guava. The result is in the line with the result of [18], they studied that by applying organic matter can improve productivity of guava orchard. [14] reported that $95 \%$ t of the orchards guava orchards in Kohat district of Pakistan were adequate in organic matter in the surface and $48 \%$ in thesubsurface. 
Table 3. Organic matter (\%) content in guava orchard soils of district Larkan, Sindh

\begin{tabular}{|c|c|c|c|c|c|c|c|}
\hline \multirow[b]{2}{*}{ Taluka } & \multirow{2}{*}{$\begin{array}{l}\text { Soil de pth } \\
\text { (cm) }\end{array}$} & \multirow[b]{2}{*}{ Range } & \multirow[b]{2}{*}{ Average } & \multirow{2}{*}{$\begin{array}{l}\text { Mean } \pm \\
\text { standard } \\
\text { de viation }\end{array}$} & \multicolumn{3}{|c|}{ Categorization (sample \%) } \\
\hline & & & & & $\begin{array}{l}\text { Poor } \\
(<0.86)\end{array}$ & $\begin{array}{l}\text { Medium } \\
(0.86-1.29)\end{array}$ & $\begin{array}{l}\text { High } \\
(>1.29\end{array}$ \\
\hline \multirow{3}{*}{ Bakrani } & $0-15$ & $0.85-1.59$ & 1.2 & $1.2 \pm 0.52$ & $10 \%$ & $70 \%$ & $20 \%$ \\
\hline & $15-30$ & $0.62-0.98$ & 0.8 & $0.8 \pm 0.25$ & $70 \%$ & $30 \%$ & - \\
\hline & $30-45$ & $0.28-0.45$ & 0.36 & $0.36 \pm 0.12$ & $100 \%$ & - & - \\
\hline \multirow{3}{*}{ Dokri } & $0-15$ & $0.68-1.13$ & 0.90 & $0.90 \pm 0.32$ & $80 \%$ & $20 \%$ & - \\
\hline & $15-30$ & $0.49-0.65$ & 0.57 & $0.57 \pm 0.11$ & $100 \%$ & - & - \\
\hline & $30-45$ & $0.25-0.50$ & 0.37 & $0.37 \pm 0.10$ & $100 \%$ & - & - \\
\hline \multirow{3}{*}{ Larkana } & $0-15$ & $0.54-0.84$ & 0.69 & $0.69 \pm 0.21$ & $100 \%$ & - & - \\
\hline & $15-30$ & $0.38-0.70$ & 0.54 & $0.54 \pm 0.23$ & $100 \%$ & - & - \\
\hline & $30-45$ & $0.22-0.56$ & 0.39 & $0.39 \pm 0.19$ & $100 \%$ & - & - \\
\hline \multirow{3}{*}{ Ratodero } & $0-15$ & $0.49-1.18$ & 0.83 & $0.83 \pm 0.49$ & $80 \%$ & $20 \%$ & - \\
\hline & $15-30$ & $0.32-0.82$ & 0.57 & $0.57 \pm 0.35$ & $100 \%$ & - & - \\
\hline & $30-45$ & $0.21-0.79$ & 0.5 & $0.5 \pm 0.41$ & $100 \%$ & - & - \\
\hline
\end{tabular}

\section{Calcium carbonate $(\%)$}

The $\mathrm{CaCO}_{3}$ content of this study was categorized according to the categorization for calcareous soils as given by Jackson [15]. All the guava orchard soils were categorized as very marginal calcareous (5$10 \%$ ). Table 4 showed that there is slightly variation in $\mathrm{CaCO}_{3}$ of soil at various depths. The values of $\mathrm{CaCO}_{3}$ of Ratodero soils were observed high at surface soils than subsurface. The lowest content was observed in Bakrani with the values ranging between 4.2 and $5.32 \%, 4.23$ and 5.43 and 4.45 and $5.75 \%$ with average of 4.64 and $5.01 \%$ at various soil depths respectively. For taluka Dokri and larkana, the $\mathrm{CaCO}_{3}$ contents were between 5.15 and $5.45 \%, 5.25$ and $5.65 \%$ and 5.46 and 5.85 $\%$. The averages contents values of 5.32, 5.66 and $6.1 \%$ respectively at three various soil depths. Similar results were reported by Mehvish et al. [14].

Table 4. Calcium carbonate $(\%)$ content in guava orchard soils of district Larkana, Sindh

\begin{tabular}{|c|c|c|c|c|c|c|c|}
\hline \multirow[b]{2}{*}{ Taluka } & \multirow{2}{*}{$\begin{array}{l}\text { Soil depth } \\
\text { (cm) }\end{array}$} & \multirow[b]{2}{*}{ Range } & \multirow[b]{2}{*}{ Average } & \multirow{2}{*}{$\begin{array}{c}\text { Mean } \pm \\
\text { standard } \\
\text { de viation }\end{array}$} & \multicolumn{3}{|c|}{ Categorization (sample \%) } \\
\hline & & & & & $\begin{array}{l}\text { Low } \\
(<5)\end{array}$ & $\begin{array}{c}\text { Marginal } \\
(5-10)\end{array}$ & $\begin{array}{c}\text { Adequate } \\
(>15)\end{array}$ \\
\hline \multirow{3}{*}{ Bakrani } & $0-15$ & $4.45-5.75$ & 5.1 & $5.1 \pm 0.02$ & $80 \%$ & $20 \%$ & - \\
\hline & $15-30$ & $4.23-5.43$ & 4.83 & $4.83 \pm 0.02$ & $80 \%$ & $20 \%$ & - \\
\hline & $30-45$ & $4.2-5.32$ & 4.76 & $4.76 \pm 0.03$ & $80 \%$ & $20 \%$ & - \\
\hline \multirow{3}{*}{ Dokri } & $0-15$ & $5.46-5.85$ & 5.65 & $5.65 \pm 0.02$ & - & $100 \%$ & - \\
\hline & $15-30$ & $5.25-5.65$ & 5.45 & $5.45 \pm 0.02$ & - & $100 \%$ & - \\
\hline & $30-45$ & $5.15-5.45$ & 5.35 & $5.35 \pm 0.01$ & - & $100 \%$ & - \\
\hline \multirow{3}{*}{ Larkana } & $0-15$ & $5.46-5.67$ & 5.56 & $5.56 \pm 0.01$ & - & $100 \%$ & - \\
\hline & $15-30$ & $5.43-5.50$ & 5.46 & $5.46 \pm 0.01$ & - & $100 \%$ & - \\
\hline & $30-45$ & $5.35-5.45$ & 5.4 & $5.4 \pm 0.01$ & - & $100 \%$ & - \\
\hline \multirow{3}{*}{ Ratodero } & $0-15$ & $7.28-7.85$ & 7.56 & $7.56 \pm 0.01$ & - & $100 \%$ & - \\
\hline & $15-30$ & $7.05-7.52$ & 7.28 & $7.28 \pm 0.01$ & - & $100 \%$ & - \\
\hline & $30-45$ & $7.01-7.35$ & 7.18 & $7.18 \pm 0.01$ & - & $100 \%$ & - \\
\hline
\end{tabular}


Soluble $\mathrm{Na}^{+}\left(\right.$meqL $\left.^{-1}\right)$ in Soil

Results regarding soluble $\mathrm{Na}^{+}$(Table 5) showed that a slightly variation was found at different soil depths in all selected Talukas of district Larkana. Average soluble $\mathrm{Na}^{+}$ contents ranged between $0.30-0.45 \mathrm{meqL}^{-1}$ across the four taluka's of Larkana. The orchard of Taluka Dokri contained more $\mathrm{Na}^{+}$ than other Taluka orchard soils. Overall results showed that $100 \%$ soil samples were found marginal in soluble $\mathrm{Na}^{+}$.
Soluble calcium (meqL $\left.\mathrm{L}^{-1}\right)$

The result of soluble $\mathrm{Ca}$ has been given in Table 6. It indicates that soluble $\mathrm{Ca}$ of four taluka's of district Larkana ranged between 5.37 to 8.74 meq $\mathrm{L}^{-1}$. Guava orchard soils of Ratodero have more Ca content as compared to other taluka's followed by Larkana. Categorization of soil samples for soluble $\mathrm{Ca}$ indicates that $100 \% \mathrm{Ca}$ content of the samples were found marginal.

Table 5. Soluble $\mathrm{Na}^{+}\left(\mathrm{meqL}^{-1}\right)$ in guava orchard s oils of fourtaluka's of dis trict Larkana, Sindh

\begin{tabular}{|c|c|c|c|c|c|c|c|}
\hline \multirow[b]{2}{*}{ Taluka } & \multirow{2}{*}{$\begin{array}{l}\text { Soil } \\
\text { depth } \\
(\mathrm{cm})\end{array}$} & \multirow[b]{2}{*}{ Range } & \multirow[b]{2}{*}{ Average } & \multirow{2}{*}{$\begin{array}{l}\text { Mean } \pm \\
\text { standard } \\
\text { deviation }\end{array}$} & \multicolumn{3}{|c|}{ Categorization (s ample \%) } \\
\hline & & & & & $\begin{array}{l}\text { Low } \\
(<0.1)\end{array}$ & $\begin{array}{l}\text { Marginal } \\
(0.3-0.7)\end{array}$ & $\begin{array}{l}\text { Adequate } \\
(>2)\end{array}$ \\
\hline \multirow{3}{*}{ Bakrani } & $0-15$ & $0.32-0.39$ & 0.35 & $0.35 \pm 0.02$ & - & $100 \%$ & - \\
\hline & $15-30$ & $0.31-0.38$ & 0.34 & $0.34 \pm 0.02$ & - & $100 \%$ & - \\
\hline & $30-45$ & $0.23-0.35$ & 0.29 & $0.29 \pm 0.03$ & - & $100 \%$ & - \\
\hline \multirow{3}{*}{ Dokri } & $0-15$ & $0.42-0.49$ & 0.45 & $0.45 \pm 0.02$ & - & $100 \%$ & - \\
\hline & $15-30$ & $0.41-0.47$ & 0.44 & $0.44 \pm 0.02$ & - & $100 \%$ & - \\
\hline & $30-45$ & $0.41-0.45$ & 0.43 & $0.43 \pm 0.01$ & - & $100 \%$ & - \\
\hline \multirow{3}{*}{ Larkana } & $0-15$ & $0.24-0.49$ & 0.36 & $0.36 \pm 0.01$ & - & $100 \%$ & - \\
\hline & $15-30$ & $0.23-0.47$ & 0.35 & $0.35 \pm 0.01$ & - & $100 \%$ & - \\
\hline & $30-45$ & $0.21-0.45$ & 0.33 & $0.33 \pm 0.01$ & - & $100 \%$ & - \\
\hline \multirow{3}{*}{ Ratodero } & $0-15$ & $0.36-0.39$ & 0.37 & $0.37 \pm 0.01$ & - & $100 \%$ & - \\
\hline & $15-30$ & $0.32-0.38$ & 0.35 & $0.35 \pm 0.01$ & - & $100 \%$ & - \\
\hline & $30-45$ & $0.31-036$ & 0.33 & $0.33 \pm 0.01$ & - & $100 \%$ & - \\
\hline
\end{tabular}

Table 6. Soluble $\mathrm{Ca}^{2+}\left(\mathrm{meq}^{-1}\right)$ in guava orchard soils of four taluka's of dis trict Larkana, Sindh

\begin{tabular}{|c|c|c|c|c|c|c|c|}
\hline \multirow[b]{2}{*}{ Taluka } & \multirow{2}{*}{$\begin{array}{l}\text { Soil } \\
\text { depth } \\
\text { (cm) }\end{array}$} & \multirow[b]{2}{*}{ Range } & \multirow[b]{2}{*}{ Average } & \multirow{2}{*}{$\begin{array}{l}\text { Mean } \pm \\
\text { standard } \\
\text { deviation }\end{array}$} & \multicolumn{3}{|c|}{ Categorization (sample \%) } \\
\hline & & & & & $\begin{array}{l}\text { Low } \\
(<1-2)\end{array}$ & $\begin{array}{l}\text { Marginal } \\
(2-10)\end{array}$ & $\begin{array}{l}\text { Adequate } \\
(>20)\end{array}$ \\
\hline \multirow{3}{*}{ Bakrani } & $0-15$ & $5.5-5.9$ & 5.7 & $5.7 \pm 0.1$ & - & $100 \%$ & - \\
\hline & $15-30$ & $5.2-5.8$ & 5.5 & $5.5 \pm 0.1$ & - & $100 \%$ & - \\
\hline & $30-45$ & $5.1-5.8$ & 5.45 & $5.45 \pm 0.2$ & - & $100 \%$ & - \\
\hline \multirow{3}{*}{ Dokri } & $0-15$ & $6.56-6.95$ & 6.75 & $6.75 \pm .0 .11$ & - & $100 \%$ & - \\
\hline & $15-30$ & $6.45-6.75$ & 6.6 & $6.6 \pm 0.11$ & - & $100 \%$ & - \\
\hline & $30-45$ & $6.22-6.56$ & 6.42 & $6.42 \pm 0.12$ & - & $100 \%$ & - \\
\hline \multirow{3}{*}{ Larkana } & $0-15$ & $7.56-7,98$ & 7.77 & $7.77 \pm 0.09$ & - & $100 \%$ & - \\
\hline & $15-30$ & $7.54-7.86$ & 7.77 & $7.77 \pm 0.01$ & - & $100 \%$ & - \\
\hline & $30-45$ & $7.22-7.55$ & 7.38 & $7,38 \pm 0.01$ & - & $100 \%$ & - \\
\hline \multirow{3}{*}{ Ratodero } & $0-15$ & $7.66-9.75$ & 8.70 & $8.70 \pm 0.86$ & - & $100 \%$ & - \\
\hline & $15-30$ & $7.54-9.65$ & 8.59 & $8.59 \pm 0.6$ & - & $100 \%$ & - \\
\hline & $30-45$ & $7.22-9.56$ & 8.39 & $8.39 \pm 0.7$ & - & $100 \%$ & - \\
\hline
\end{tabular}

Soluble magnesium (meq $\left.\mathbf{L}^{-1}\right)$

The result of soil $\mathrm{Mg}$ content (Table 7) in guava orchard of four taluka revealed that
$100 \%$ soil samples were found marginal. No any single soil sample was found low or adequate in $\mathrm{Mg}$ content. Comparatively, 
Bakrani orchard soils had higher $\mathrm{Mg}$ than other taluka's. Overall $\mathrm{Mg}$ content ranged between 2.5 to 3.7 meq $\mathrm{L}^{-1}$ in all guava orchards of the district.

Table 7. Soluble $M g\left(\mathrm{meq}^{-1}\right)$ in guava orchard soils of four taluka's of dis trict Larkana, Sindh

\begin{tabular}{|c|c|c|c|c|c|c|c|}
\hline \multirow[b]{2}{*}{ Taluka } & \multirow{2}{*}{$\begin{array}{l}\text { Soil } \\
\text { depth } \\
\text { (cm) }\end{array}$} & \multirow[b]{2}{*}{ Range } & \multirow[b]{2}{*}{ Average } & \multirow{2}{*}{$\begin{array}{l}\text { Mean } \pm \\
\text { standard } \\
\text { de viation }\end{array}$} & \multicolumn{3}{|c|}{ Categorization (sample \%) } \\
\hline & & & & & $\begin{array}{l}\text { Low } \\
(<0.5)\end{array}$ & $\begin{array}{l}\text { marginal } \\
(1-3)\end{array}$ & $\begin{array}{l}\text { Ade quate } \\
(>7)\end{array}$ \\
\hline \multirow{3}{*}{ B akrani } & $0-15$ & $3.56-3.99$ & 3.77 & $3.77 \pm 0.15$ & - & $100 \%$ & - \\
\hline & $15-30$ & $3.35-3.77$ & 3.56 & $3.56 \pm 0.15$ & - & $100 \%$ & - \\
\hline & $30-45$ & $3.25-3.66$ & 3.45 & $3.45 \pm 0.13$ & - & $100 \%$ & - \\
\hline \multirow{3}{*}{ Dokri } & $0-15$ & $2.29-2.98$ & 2.63 & $2.63 \pm 0.2$ & - & $100 \%$ & - \\
\hline & $15-30$ & $2.26-2.85$ & 2.55 & $2.55 \pm 0.1$ & - & $100 \%$ & - \\
\hline & $30-45$ & $2.22-2.55$ & 2.38 & $2.38 \pm 0.3$ & - & $100 \%$ & - \\
\hline \multirow{3}{*}{ Larkana } & $0-15$ & $2.29-3.67$ & 2.98 & $2.98 \pm 0.1$ & - & $100 \%$ & - \\
\hline & $15-30$ & $2.26-3.58$ & 2.92 & $2.92 \pm 0.1$ & - & $100 \%$ & - \\
\hline & $30-45$ & $2.2-3.57$ & 2.88 & $2.88 \pm 0.1$ & - & $100 \%$ & - \\
\hline \multirow{3}{*}{ Ratodero } & $0-15$ & $2.35-3.78$ & 3.06 & $3.06 \pm 0.01$ & - & $100 \%$ & - \\
\hline & $15-30$ & $2.24-3.68$ & 2.96 & $2.96 \pm 0.01$ & - & $100 \%$ & - \\
\hline & $30-45$ & $2.15-3.58$ & 2.86 & $2.86 \pm 0.00$ & - & $100 \%$ & - \\
\hline
\end{tabular}

\section{Soluble $\mathbf{K}^{+}\left(\operatorname{meq} \mathbf{L}^{-1}\right)$ in Soil}

Potassium content in soil of selected orchards is presented in Table 8. This table shows that $\mathrm{K}^{+}$ranged between $302-403$ with an average $\mathrm{K}^{+}$of $351 \mathrm{meqL}^{-1}$ across four talukas. Maximum $\mathrm{K}^{+}$as recorded in the soil of Larkana orchard followed by Bakrani. However, Ratodero had relatively lower values of $\mathrm{K}^{+}$. In general, result of $\mathrm{K}^{+}$ showed that $100 \%$ soils were found above adequate level. Similar results are reported by Mehvish et al. [14], who assessed fertility status of the soils of 44 guava orchards in Kohat District. They found that Available K was between 103 and $393 \mathrm{mg}$ $\mathrm{kg}^{-1}$, Potassium was marginal in $16 \%$ and high in $84 \%$.

Table 8. Soluble $\mathrm{K}^{+}\left(\operatorname{meq~} \mathrm{L}^{-1}\right)$ in guava orchard soils of four Talukas of dis trict Larkana Sindh

\begin{tabular}{|c|c|c|c|c|c|c|c|}
\hline \multirow[b]{2}{*}{ Taluka } & \multirow{2}{*}{$\begin{array}{l}\begin{array}{l}\text { Soil } \\
\text { depth } \\
\text { (cm) }\end{array} \\
\end{array}$} & \multirow[b]{2}{*}{ Range } & \multirow[b]{2}{*}{ Average } & \multirow{2}{*}{$\begin{array}{l}\text { Mean } \pm \\
\text { standard } \\
\text { de viation }\end{array}$} & \multicolumn{3}{|c|}{ Cate gorization (sample \%) } \\
\hline & & & & & $\begin{array}{l}\text { Low } \\
(<60)\end{array}$ & $\begin{array}{l}\text { Marginal } \\
(60-120)\end{array}$ & $\begin{array}{l}\text { Adequate } \\
(>120)\end{array}$ \\
\hline \multirow{3}{*}{ Bakrani } & $0-15$ & $260-500$ & 380 & $380 \pm 169.7$ & - & - & $100 \%$ \\
\hline & $15-30$ & $300-532$ & 416 & $416 \pm 156.9$ & - & - & $100 \%$ \\
\hline & $30-45$ & $180-470$ & 325 & $325 \pm 205.1$ & - & - & $100 \%$ \\
\hline \multirow{3}{*}{ Dokri } & $0-15$ & $180-560$ & 370 & $370 \pm 268.7$ & - & - & $100 \%$ \\
\hline & $15-30$ & $260-510$ & 385 & $385 \pm 176.8$ & - & - & $100 \%$ \\
\hline & $30-45$ & $180-520$ & 350 & $350 \pm 240.4$ & - & - & $100 \%$ \\
\hline \multirow{3}{*}{ Larkana } & $0-15$ & $250-560$ & 405 & $405 \pm 212.1$ & - & - & $100 \%$ \\
\hline & $15-30$ & $260-510$ & 385 & $385 \pm 134.3$ & - & - & $100 \%$ \\
\hline & $30-45$ & $180-470$ & 325 & $325 \pm 162.6$ & - & - & $100 \%$ \\
\hline \multirow{3}{*}{ Ratodero } & $0-15$ & $190-510$ & 350 & $350 \pm 134.3$ & - & - & $100 \%$ \\
\hline & $15-30$ & $200-440$ & 320 & $320 \pm 49.5$ & - & - & $100 \%$ \\
\hline & $30-45$ & $210-500$ & 355 & $355 \pm 56.6$ & - & - & $100 \%$ \\
\hline
\end{tabular}


Soluble $\mathrm{Cl}^{-}\left(\operatorname{meq} \mathbf{L}^{-1}\right)$

Results for soluble $\mathrm{Cl}^{-}$(Table 9) describes that a slightly variation at various soils depth among all taluka larkana was recorded. An average soluble $\mathrm{Cl}^{-}$content ranged between
0.1-0.3 meqL $\mathrm{L}^{-1}$. Ratodero taluka orchards have little more $\mathrm{Cl}^{-}$than other taluka orchards soils. Overall results revealed that $100 \%$ soil samples were observed low in $\mathrm{Cl}^{-}$ content.

Table 9. Soluble $\mathrm{Cl}^{-}\left(\right.$meqL $\left.^{-1}\right)$ in guava orchard soils of four Talukas of District Larkana, Sindh

\begin{tabular}{|c|c|c|c|c|c|c|c|}
\hline \multirow[b]{2}{*}{ Taluka } & \multirow{2}{*}{$\begin{array}{l}\text { Soil } \\
\text { depth } \\
\text { (cm) }\end{array}$} & \multirow[b]{2}{*}{ Range } & \multirow[b]{2}{*}{ Average } & \multirow{2}{*}{$\begin{array}{l}\text { Mean } \pm \\
\text { standard } \\
\text { de viation }\end{array}$} & \multicolumn{3}{|c|}{ Categorization (sample \%) } \\
\hline & & & & & $\begin{array}{l}\text { Low } \\
(<4)\end{array}$ & $\begin{array}{l}\text { Marginal } \\
(4-10)\end{array}$ & $\begin{array}{l}\text { Adequate } \\
(>10)\end{array}$ \\
\hline \multirow{3}{*}{ Bakrani } & $0-15$ & $0.02-0.056$ & 0.03 & $0.03 \pm 0.01$ & $100 \%$ & - & - \\
\hline & $15-30$ & $0.01-0.054$ & 0.02 & $0.02 \pm 0.01$ & $100 \%$ & - & - \\
\hline & $30-45$ & $0.001-0.032$ & 0.01 & $0.01 \pm 0,00$ & $100 \%$ & - & - \\
\hline \multirow{3}{*}{ Dokri } & $0-15$ & $0.001-0.04$ & 0.01 & $0.01 \pm 0.01$ & $100 \%$ & - & - \\
\hline & $15-30$ & $0.001-0.03$ & 0.01 & $0.01 \pm 0.01$ & $100 \%$ & - & - \\
\hline & $30-45$ & $0.0001-0.03$ & 0.01 & $0.01 \pm 0.01$ & $100 \%$ & - & - \\
\hline \multirow{3}{*}{ Larkana } & $0-15$ & $0.001-0.054$ & 0.01 & $0.01 \pm 0.01$ & $100 \%$ & - & - \\
\hline & $15-30$ & $0.015-0.042$ & 0.01 & $0.01 \pm 0.01$ & $100 \%$ & - & - \\
\hline & $30-45$ & $0.001-0.032$ & 0.001 & $0.001 \pm 0.01$ & $100 \%$ & - & - \\
\hline \multirow{3}{*}{ Ratodero } & $0-15$ & $0.025-0.056$ & 0.03 & $0.03 \pm 0.00$ & $100 \%$ & - & - \\
\hline & $15-30$ & $0.015-0.054$ & 0.029 & $0.29 \pm 0.01$ & $100 \%$ & - & - \\
\hline & $30-45$ & $0.01-0.052$ & 0.02 & $0.02 \pm 0.01$ & $100 \%$ & - & - \\
\hline
\end{tabular}

\section{Conclusions}

It is concluded from above results that the soils of guava orchards of district Larkana are generally heavy in texture, non-saline, strongly alkaline and poor in organic matter, moderately to medium calcareous in nature. The soil was found marginal in soluble $\mathrm{Na}$, $\mathrm{Ca}$, Mg. The available potassium was quite adequate. Therefore, the application of organic matterand the macro nutrients fertilizers are suggested to maintain soil fertility as well as yield of the guava in the guava orchards.

\section{Authors' contributions}

Conceived and designed the experiments: AW Gandahi \& M Memon, Performed the Experiments: M Mukhtar, A Ali \& S Hussain, Analyzed the Data: M Mukhtar \& M Kumar \& M Kumar, Wrote the paper: A Ali \& M Kumar.

\section{References}

1. Tarun A, Kumar K, Singha A, Shukla SK \& Singh VK (2014). Assessing soil characteristics and guava orchard productivity as Influenced by organic and inorganic substrates. J Anim Plant Sci 24(4): 1157-1165.

2. Takamitsu K, Mukai M, Kiwako S, Araki, Adhikari D \& Kubo M (2016). Analysis of chemical and biological soil properties in organically and conventionally fertilized apple orchards. J Agrl Chem Environ (5): 92-99.

3. Tejada M, Hernandez MT \& Garcia C (2006). Application of two organic amendments on soil restoration: Effects on the soil biological properties. $J$ Environ Qual 35: 1010-1017.

4. Shakeel, A, Aslam HKW, Shoaib M, Sikandar HA \& Ramzan R (2013). Effect of various hydrocolloids on cloud stability and nutrition of carrot juice. $J$ Glob. Innov Agric Soc Sci 1: 22-27.

5. MINFAL (2006-2007). Agricultural statistics of Pakistan. Government of Pakistan. Ministry of food and 
Agriculture and Livestock (Economic wing) pp. 331-339.

6. GoP (2012). Agricultural statistics of Pakistan, 2010 - 2011. Statistics (1) Trison, Pakistan Bureau of statistics, Islamabad. pp. 272.

7. Bal JS \& Dhaliwal GS (2004). Distribution and quality characteristics of graded guava fruits. Haryana J Hort Sci 33: 53-54.

8. Zang XP \& Tao LX (2000). The application of fertilizer for guava trees. $J$ South China Fruits 29(6): 29-31.

9. Bhatnagar P \& Chandra A (2003). Nutritional survey of guava orchards in biknaner district. Curro Agric 27: 133135.

10. Divya \& Kumari A (2009). Effect of different temperatures, timings and storage periods on the physic-chemical and nutritional characteristics of Whey Guava beverage. World J Dairy Food Sci 4: 118-122.

11. GoP (2010). Fruits, vegetables and condiments statistics of Pakistan. Government of Pakistan, Ministry of Food, Agriculture and Livestock (economic wing) Islambad.

12. Natale W (1997). Goiabeira: Extração de nutrientes pela poda. In:
Simpósiobrasileirosobreacultura da goibeira, 1. Jaboticabal, 1997. Anais. Jaboticabal: FCAV/UNESP.

13. Natale W, Coutinho ELM, Pereira \& Boaretto AE (2002). Nutrients foliar content for higher production cultivars of guava in Brazil. Acta Hort 594: 383386.

14. Mehvish F, Khan S, Razzaq A, Nawaz I \& Haq I (2011). Fertility status of guava orchards in Kohat District of Pakistan. $J$ Soil Sci Environ Manage 3(9): 260-268.

15. Jackson ML (1962). Soil chemical analysis. Constable Co. Ltd., London.

16. Walkley A \& Black IA (1934). An examination of the Degtjareff method for determining soil organic matter, and a proposed modification of the chromic acid titration method. Soil Sci 37: 29-38.

17. Faqir MM, Shakir A \& Salik MR (2000). Effect of individual combined application of organic and inorganic manures on the productivity of guava (Psidium guajava L.)

18. Ishtiaq H, Khurshid W \& Iqbal $\mathrm{K}$ (2012). Factors responsible for decline in guava (psidiumguajava) yield. J Agric Res 50(1): 129-134. 